\title{
First-principles investigations of the orbital magnetic moments in $\mathrm{CrO}_{2}$
}

\author{
Horng-Tay Jeng ${ }^{\text {a) }}$ \\ Physics Division, National Center for Theoretical Sciences, Hsinchu 30043, Taiwan \\ G. Y. Guo \\ Department of Physics, National Taiwan University, Taipei 10617, Taiwan
}

(Received 14 December 2001; accepted for publication 24 April 2002)

\begin{abstract}
The electronic and magnetic properties of the ferromagnetic half-metal $\mathrm{CrO}_{2}$ have been studied by using the full-potential linear muffin-tin orbital method within the local-spin-density approximation (LSDA) and the LSDA $+U$ approach. The orbital magnetic moments are investigated by including the spin-orbit coupling in both schemes. Compared with the orbital contributions to magnetization in other transition metal oxides, the orbital magnetic moment of the $\mathrm{Cr}$ atom is quenched, while the $\mathrm{O}$ atoms exhibit a relatively significant orbital moment in $\mathrm{CrO}_{2}$. The LSDA gives the orbital moments of $-0.037 \mu_{B} / \mathrm{Cr}$ and $-0.0011 \mu_{B} / \mathrm{O}$. They are too small as compared with the moments of $-0.05 \mu_{B} / \mathrm{Cr}$ and $-0.003 \mu_{B} / \mathrm{O}$ obtained from $\mathrm{x}$-ray magnetic circular dichroism measurements. By taking the on-site Coulomb energy $U$ into account, both the spin and orbital magnetic moments of $\mathrm{Cr}$ and $\mathrm{O}$ are dramatically enhanced. For a Hubbard $U$ of $3 \mathrm{eV}$, the LSDA $+U$ gives orbital moments of $-0.051 \mu_{B} / \mathrm{Cr}$ and $-0.0025 \mu_{B} / \mathrm{O}$, in good agreement with the experimental orbital moments. Both the spin and orbital moments are found to increase more or less linearly with increasing U. Similar trends have also been found in $\mathrm{CoO}$ and $\mathrm{NiO}$. (C) 2002 American Institute of Physics. [DOI: $10.1063 / 1.1486260]$
\end{abstract}

\section{INTRODUCTION}

Transition metal oxides have been found to exhibit diverse physical properties such as strong Coulomb correlations, strong spin and orbital fluctuations, mass renormalization effects, incoherence of charge dynamics, and phase transitions. ${ }^{1}$ They were investigated extensively in the past decade because of the interesting fundamental physics as well as the important technological applications. The localspin-density approximation (LSDA) has been successfully used in electronic structure calculations for many materials. However, for the $3 d$ transition metal oxides, the $3 d$ electrons are in the relatively localized Wannier states rather than in the itinerant Bloch states. Hence the on-site $3 d$ electronelectron Coulomb interaction is fairly strong such that the LSDA band calculations are often not adequate for these strongly correlated systems. In many cases, the LSDA gives moments and gaps which are too small, or even gives no gap for the Mott insulators. ${ }^{2}$ By taking the on-site Coulomb interaction energy $U$ into account, the LSDA $+U$ method successfully improves the LSDA results for many transition metal oxides. ${ }^{3}$

$\mathrm{CrO}_{2}$ is a ferromagnetic half-metal with a magnetic moment of $2 \mu_{B}$ per formula unit (fu). ${ }^{4}$ It has been widely used in magnetic recording tapes. In recent years, it has attracted substantial interest because of its half-metallic property and its applications in spintronics. In half-metal $\mathrm{CrO}_{2}$, one spin channel is metallic and the other is insulating, resulting in an unusual transport property of $100 \%$ spin polarization. The Fermi level lies in the partially filled $3 d$ band of the majority

\footnotetext{
a) Author to whom correspondence should be addressed; electronic mail: jeng@phys.nthu.edu.tw
}

spin, whereas in the minority spin, the Fermi energy falls in an exchange split gap between the occupied oxygen $2 p$ band and the unoccupied chromium $3 d$ band. Since the states involving the conduction and magnetic properties are highly spin-polarized near the Fermi energy, it is interesting to investigate the orbital contributions of individual atoms to the magnetic moment of $\mathrm{CrO}_{2}$.

$\mathrm{CrO}_{2}$ crystallizes in the tetragonal rutile structure with two formula units per unit cell (Fig. 1). The $\mathrm{Cr}$ atoms form a body-centered tetragonal lattice. Each $\mathrm{Cr}$ atom is surrounded by a distorted oxygen octahedron with the octahedron at the body's center and at the corner position differing by a $90^{\circ}$ rotation about the c-axis. In the local coordinate system for every octahedron, in which the $z$ axis is pointed to the apical oxygen and the $x$ and $y$ axes are directed to the basal plane (i.e., the crystal (1 $\overline{1} 0)$ plane) oxygens, the octahedral ligand field splits the $\mathrm{Cr}-3 d$ manifold into three lower lying $t_{2 g}$ states $\left(d_{x y}, d_{y z}\right.$, and $\left.d_{z x}\right)$ and two $e_{g}$ states $\left(d_{x^{2}-y^{2}}\right.$, and $d_{3 z^{2}-r^{2}}$ ) of higher energy. This ligand field splitting results from the hybridization between the $\mathrm{Cr} e_{g}$ states and two of the three $\mathrm{O}-2 p$ orbitals. The formation of the lower lying $\sigma$ bonding states results in the wide $2 p$ band, whereas the $e_{g}$ band energy is raised by the antibonding. The distortion of the octahedron further splits the $t_{2 g}$ states into localized $d_{x y}$ state and extended $d_{y z \pm z x}$ states. The extended $d_{y z \pm z x}$ states hybridize with one of the three O-2p orbitals forming the $d$ $p \pi$ bond. The lower lying occupied bonding state $d_{y z+z x}$ and the localized $d_{x y}$ state thus form the main $3 d$ peak below the Fermi level, whereas the antibonding state $d_{y z-z x}$ is pushed upwards and leaves a pseudogap at the Fermi level in the majority spin. The exchange splitting between the majority and the minority spin $\mathrm{Cr}-3 d$ bands is a consequence of the ferromagnetic phase of $\mathrm{CrO}_{2}$. This exchange splitting 


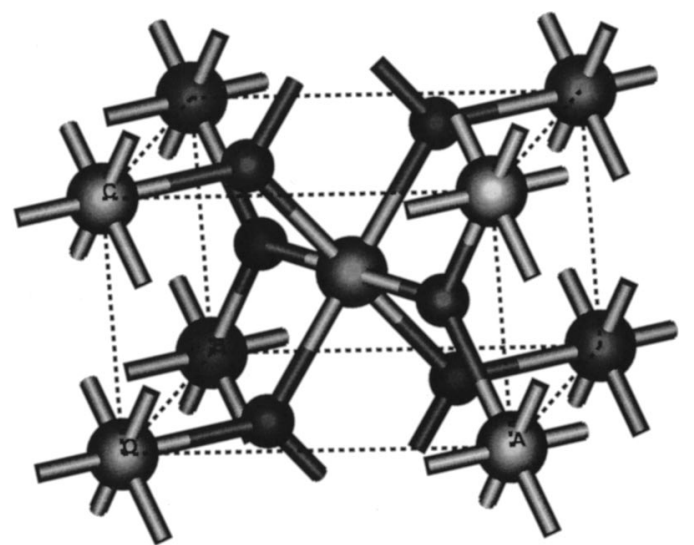

FIG. 1. Rutile structure of $\mathrm{CrO}_{2}$. Big light balls denote $\mathrm{Cr}$ atoms and small dark balls denote $\mathrm{O}$ atoms.

shifts the minority spin $3 d$ band above the Fermi level and yields a gap at the Fermi level, resulting in a half-metallic band structure (Figs. 2, 3, and 4).

The orbital magnetic moments are usually suppressed in the $3 d$ transition metals because of the crystal field in solids. Some $3 d$ transition metal oxides exhibit large unquenched orbital magnetic moments. The unquenched orbital moment

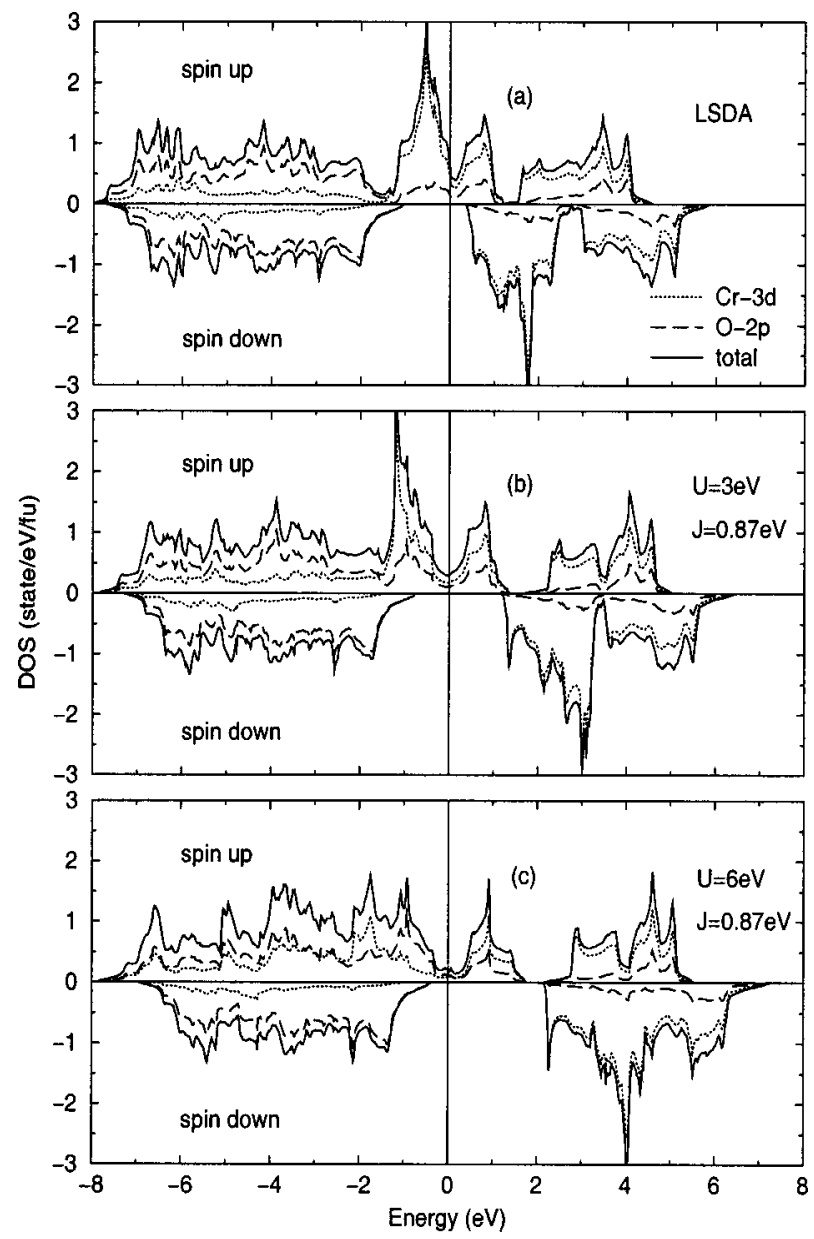

FIG. 2. Spin and orbital decomposed DOS of $\mathrm{CrO}_{2}$ from the LSDA (a) and from the LSDA $+U$ with $J=0.87 \mathrm{eV}$ and $U=3 \mathrm{eV}$ (b) and $U=6 \mathrm{eV}$ (c). The dotted, dashed, and solid lines are $\mathrm{Cr}-3 d, \mathrm{O}-2 p$, and total DOS, respectively. The Fermi level is at the zero energy.
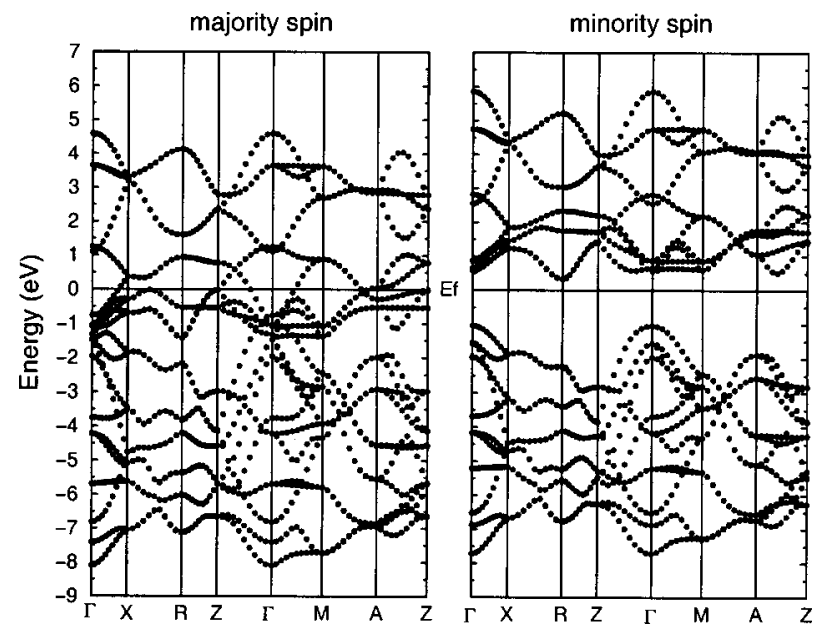

FIG. 3. Band structure of $\mathrm{CrO}_{2}$ from the LSDA. The Fermi level is at the zero energy.

arises mainly from the spin-orbit interaction in the localized $3 d$ orbital where the atomic field is deformed in a relatively slight manner by the crystal field. The strong Coulomb correlations further localize the $3 d$ orbitals and suppress the ligand field on the metal atoms. These materials are thus expected to possess larger unquenched orbital moments. It has been found in neutron-diffraction experiment for $\mathrm{CoO},{ }^{5}$ and in magnetic $\mathrm{x}$-ray scattering experiment for $\mathrm{NiO},{ }^{6}$ that the unquenched orbital magnetic moments are rather large. Although the calculated orbital moments from the LSDA are usually too small compared with experimental results, the on-site Coulomb energy $U$ in the LSDA $+U$ calculations significantly enhances the orbital moments in both $\mathrm{CoO}^{7}$ and $\mathrm{NiO},{ }^{8}$ and gives large orbital moments in agreement with experimental moments.

Several experimental techniques, such as neutron scattering, magnetic x-ray scattering, and magnetic circular dichroism in X-ray absorption (XMCD), have been proved to be useful in studying orbital magnetic moments of materials. By using sum rules, XMCD provides a powerful experimental method to deduce element-specific orbital and spin magnetic
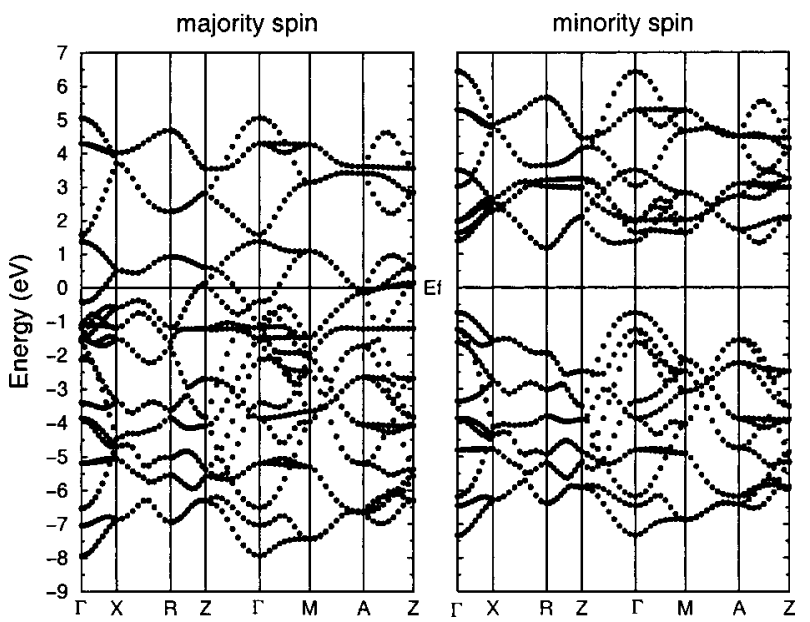

FIG. 4. Band structure of $\mathrm{CrO}_{2}$ from the $\mathrm{LSDA}+U$ with $U=3 \mathrm{eV}$ and $J$ $=0.87 \mathrm{eV}$. The Fermi level is at the zero energy. 
moments. Recently, XMCD experiments for $\mathrm{CrO}_{2}$ were performed using the elliptically polarized undulator beamline of the Synchrotron Radiation Research Center in Taiwan. ${ }^{9}$ The XMCD measurements reveal that the $\mathrm{O}-2 p$ and the $\mathrm{Cr}-3 d$ electrons are strongly hybridized. The orbital magnetic moment of $\mathrm{Cr}$ in $\mathrm{CrO}_{2}$ is quenched, while the oxygen atoms exhibit a significant orbital contribution to its magnetization, as compared with other transition metal oxides. The orbital magnetic moments of $\mathrm{Cr}$ and $\mathrm{O}$ are found to be -0.05 and $-0.003 \mu_{B}$ /atom, respectively. ${ }^{9}$ The results also show that the spin moment of $\mathrm{O}$ is antiferromagnetically coupled to that of $\mathrm{Cr}$, whereas the orbital moment is ferromagnetically coupled to that of $\mathrm{Cr}$.

The electronic band structure of $\mathrm{CrO}_{2}$ has been calculated self-consistently within the framework of the LSDA by several authors. Schwarz ${ }^{10}$ first predicted a half-metallic band structure with a spin moment of $2 \mu_{B} / \mathrm{fu}$ for $\mathrm{CrO}_{2}$. He used the augmented spherical wave (ASW) method. From the ionic viewpoint, $2 \mu_{B} / \mathrm{fu}$ is a natural consequence of Hund's rule for the $\mathrm{Cr}^{+4}\left(3 d^{2}\right)$ ion. The results of both the band theory and the ionic model agree with the experimental moment. ${ }^{4}$ Using a plan-wave pseudopotential method, Lewis et al. ${ }^{11}$ investigated the energy bands and transport properties. They put $\mathrm{CrO}_{2}$ into the category of "bad metals," in common with the high- $T_{c}$ superconductors, the high- $T$ metallic phase of $\mathrm{VO}_{2}$, and the ferromagnet $\mathrm{SrRuO}_{3}$. In the past decade, the LSDA $+U$ scheme was developed and applied to various transition metal oxides. ${ }^{3}$ Compared with the conventional LSDA, the LSDA $+U$ gives rather good results for these strongly correlated systems. Taking the on-site Coulomb energy $U$ into account (LSDA $+U$ ), Korotin et al. ${ }^{12}$ explained the conductivity in spite of the large Coulomb interactions, and concluded that $\mathrm{CrO}_{2}$ is a negative charge transfer gap material which leads to self-doping. The main Cr-3 $d$ peak above the Fermi level from the LSDA $+U$ band structure calculations agrees quite well with recent photoemission spectroscopy and specific heat measurements. ${ }^{13}$ The LSDA $+U$ model for $\mathrm{CrO}_{2}$ as a half-metallic ferromagnet with a large exchange-splitting energy, is further supported by the polarization-dependent $\mathrm{x}$-ray absorption spectroscopy and $\mathrm{x}$-ray magnetic circular dichroism measurements. ${ }^{14}$ On the other hand, Mazin et al. ${ }^{15}$ performed all-electron band-structure calculations of the transport and optical properties of $\mathrm{CrO}_{2}$ by using the full-potential linearized augmented plan-wave method (FLAPW) within the LSDA and also the generalized gradient approximation (GGA). They concluded that strong electron-magnon scattering instead of strong correlation is responsible for the renormalization of the electron bands.

\section{COMPUTATIONAL DETAILS}

In this work, the all-electron full-potential linear muffintin orbital (FP-LMTO) method ${ }^{16}$ including the spin-orbit interaction was used to perform self-consistent band structure calculations within both the LSDA and LSDA $+U^{17}$ schemes. The Vosko-Wilk-Nusair ${ }^{18}$ exchange-correlation potential and the experimental lattice parameters $a=4.419$ $\AA, c=2.912$, $\AA$ and $u=0.303^{19}$ of the rutile structure (space group $D_{4 h}^{14}: P 4_{2} / m n m$, No. 136) of $\mathrm{CrO}_{2}$ were used in the calculations. The two chromium atoms are located at the positions $(0,0,0)$, and $(1 / 2,1 / 2,1 / 2)$, and the four oxygen atoms are at $(u, u, 0),(1-u, 1-u, 0),(1 / 2+u, 1 / 2-u, 1 / 2)$, and $(1 / 2-u, 1 / 2+u, 1 / 2)$. The radii of the muffin-tin spheres used for $\mathrm{Cr}$ and $\mathrm{O}$ were $2.0 a_{0}$ and $1.5 a_{0}$, respectively. For the valence bands, a triple- $\kappa$ LMTO basis set expanded in spherical harmonics up to angular momentum $l=6$ was used for the charge densities and potentials inside the nonoverlapping muffin-tin spheres. While in the interstitial region, 7558 plane waves with energies up to 111 Ry were employed in the calculations. For the [001] direction of magnetization, the number of $k$ points used in the self-consistent calculations was 126 over the irreducible wedge of the tetragonal Brillouin zone. For the [100] magnetization, $250 \mathrm{k}$ points were used for the self-consistent calculations of lower symmetry. For simplicity, the spin and orbital magnetic moments will always be referred to [001] direction of magnetization in the rest of this paper unless stated otherwise. To explore the effects of the on-site Coulomb energy $U$ on the electronic structures and the magnetic moments, different $U$ from $2 \mathrm{eV}$ up to $9 \mathrm{eV}$ were used in the rotationally invariant LSDA $+U$ calculations ${ }^{17}$ with the exchange interaction parameter $J$ $=0.87 \mathrm{eV}^{12}$ unchanged.

\section{RESULTS AND DISCUSSION}

Figure 2 shows the spin- and orbital-decomposed density of states (DOS) of ferromagnetic $\mathrm{CrO}_{2}$ from the LSDA [Fig. 2(a)], and from the LSDA $+U$ [Figs. 2(b) and 2(c)] with exchange interaction parameter $J=0.87 \mathrm{eV}$ and on-site Coulomb energies $U=3 \mathrm{eV}$ and $U=6 \mathrm{eV}$, respectively. The LSDA results [Fig. 2(a)] agree well with earlier calculations. ${ }^{11,10,15}$ For the majority spin, the Fermi level lies near a local minimum of the $\mathrm{Cr} 3 d-t_{2 g}$ band with the DOS at the Fermi level $N\left(E_{f}\right)=0.62$ states/eV/fu. This value is close to 0.69 states/eV/fu from the pseudopotential calculations ${ }^{11}$ and smaller than 0.95 states/eV/fu from the FLAPW calculations. ${ }^{15}$ For the minority spin, the Fermi level falls in a gap of $1.34 \mathrm{eV}$, in good agreement with reported results. ${ }^{11,10,15}$ The exchange splitting between the majority and the minority spin main peaks of $\mathrm{Cr} 3 d-t_{2 g}$ bands is found to be $2.3 \mathrm{eV}$. A similar splitting of $2.5 \mathrm{eV}$ has been found in the FLAPW calculations, ${ }^{15}$ while smaller splittings of about $1.8 \mathrm{eV}$ was obtained by the $\mathrm{ASW}^{10}$ and the pseudopotential method. ${ }^{11}$ Nevertheless, all these exchange splittings obtained from different methods based on the LSDA are too small compared with the measured large splitting of about $5 \mathrm{eV}$ between the main peaks in the occupied and the empty Cr-3d DOS from photoemission spectroscopy experiments. ${ }^{13}$

Korotin et al. ${ }^{12}$ interpreted the large deviation between the LSDA and the experimental splittings as an indication of strong Coulomb correlations. They performed the LSDA $+U$ calculations with an on-site $d-d$ Coulomb energy $U=3 \mathrm{eV}$ and an exchange interaction parameter $J=0.87 \mathrm{eV}$. They used the LMTO method with the atomic-sphere approximation (LMTO-ASA). Although the Hubbard repulsion $U$ used for $\mathrm{CrO}_{2}$ is not large compared with the band width, the 
TABLE I. Majority spin DOS at the Fermi energy $N\left(E_{f}\right)$ (state/eV/fu) and minority spin band gap (eV) of $\mathrm{CrO}_{2}$ from LSDA $(U=0 \mathrm{eV})$ and LSDA $+U(U=2,3,4,6$, and $9 \mathrm{eV}, J=0.87 \mathrm{eV})$.

\begin{tabular}{lccccccc}
\hline \hline$U(\mathrm{eV})$ & 0 & 2 & 3 & 4 & 6 & 9 \\
\hline$N\left(E_{f}\right)$ & $\uparrow$ & 0.62 & 0.35 & 0.30 & 0.27 & 0.21 & 0.01 \\
$\mathrm{Gap}(\mathrm{eV})$ & $\downarrow$ & 1.34 & 1.63 & 1.90 & 2.13 & 2.49 & 3.04 \\
\hline \hline
\end{tabular}

position of the unoccupied minority spin $\mathrm{Cr}-3 d$ band is pushed up, thus resulting in an exchange splitting of about $4.5 \mathrm{eV}$, a value being compatible with experimental splitting of about $5 \mathrm{eV}^{13}$ Figure 2(b) shows our FP-LMTO results with $U=3 \mathrm{eV}$ and $J=0.87 \mathrm{eV}$ taken into account. Interestingly, below the Fermi level, the FP-LMTO density of states agree quite well with the LMTO-ASA results, ${ }^{12}$ in spite of the atomic-sphere approximation used in Ref. 12. As shown in Fig. 2(b), the main occupied $\mathrm{Cr} 3 d-t_{2 g}$ peak, which is attributed to the strongly localized $d_{x y}$ state, is located at $1.21 \mathrm{eV}$ below the Fermi level. The majority spin DOS at the Fermi level is suppressed to 0.30 states/eV/fu. The upper edge of the minority spin occupied $\mathrm{O}-2 p$ band is $0.73 \mathrm{eV}$ below the Fermi level. Above the Fermi level, there are some discrepancies between the LMTO-ASA and the FP-LMTO results, especially in the $e_{g}$ bands. However the deviations in the $t_{2 g}$ bands are not very significant. The lower edge and the main peak of the minority spin unoccupied $t_{2 g}$ band from the FP-LMTO calculations with $U=3 \mathrm{eV}$ and $J=0.87 \mathrm{eV}$ [Fig. 2(b)] are 1.17 and $3.01 \mathrm{eV}$ above the Fermi level, respectively. The resultant gap in the minority spin and the exchange splitting between the majority and the minority spin $t_{2 g}$ bands are thus 1.90 and $4.22 \mathrm{eV}$, respectively, being slightly smaller than 2.3 and $4.5 \mathrm{eV}$ from the LMTO-ASA calculations, ${ }^{12}$ respectively.

It can be seen from Fig. 2 that the on-site Coulomb energy $U$ dramatically affects the DOS of $\mathrm{CrO}_{2}$. As $U$ increases, the occupied majority spin $\mathrm{O}-2 p, \mathrm{Cr}-3 d_{x y}$, and $\mathrm{Cr}-$ $3 d_{y z+z x}$ bands are shifted downwards, while the occupied minority spin O-2p band is shifted upwards. A direct consequence of these shifts is an increase in the spin moment with respect to increasing $U$. The main occupied $\mathrm{Cr}-3 d_{x y}$ peak at $0.53 \mathrm{eV}$ below the Fermi energy given from the LSDA is shifted to $1.21 \mathrm{eV}$ below the Fermi level with $U=3 \mathrm{eV}$, which agrees well with the photoemission spectroscopy measurement. ${ }^{13}$ Since the main occupied Cr-3 $d_{x y}$ peak below the Fermi energy are sensitive to the magnitude of $U$, they are shifted further away from the Fermi level for larger $U$. Besides the shifts of the occupied $\mathrm{Cr}-3 d_{x y}$ and $\mathrm{Cr}-3 d_{y z+z x}$ bands towards lower energy, the unoccupied $\mathrm{Cr}-3 d_{y z-z x}$ band is shifted to higher energy with increasing $U$. Hence the pseudogap in the majority spin DOS at the Fermi level becomes more significant. As shown in Fig. 2, there is a tendency to open a gap at the Fermi level for larger values of $U$. The majority spin DOS at the Fermi energy $N\left(E_{f}\right)$ and the minority spin band gap between the occupied O-2 $p$ band and the unoccupied $\mathrm{Cr}-3 d$ band for different $U$ are listed in Table I. For $U=9 \mathrm{eV}$, a gap in the majority spin is almost open with $N\left(E_{f}\right)=0.01$ states/eV/fu only, which would make $\mathrm{CrO}_{2}$ a Mott-Hubbard insulator. For higher values of $U$, the unoccupied majority spin $e_{g}$ band and the minority spin $t_{2 g}$ and $e_{g}$ bands are further shifted towards higher energy, leading to larger band gaps and exchange splittings.

Figures 3 and 4 show, respectively, the FP-LMTO band structures (BS) of $\mathrm{CrO}_{2}$ from the LSDA and from the LSDA $+U$ with $U=3 \mathrm{eV}$ and $J=0.87 \mathrm{eV}$. They agree well with the reported results. ${ }^{11,10,15,12}$ The strongly localized $\mathrm{Cr}$ $3 d_{x y}$ states from the LSDA $+U$ are shown in the almost dispersionless majority spin band at about $1.2 \mathrm{eV}$ below the Fermi energy (Fig. 4). It is found that $\mathrm{CrO}_{2}$ is a near itinerant material in which the Coulomb correlations are not very strong as compared with those in other transition metal oxides. Note that the GGA has also been used to calculate the DOS and BS in this work. The GGA gives an accurate lattice constant of $a=4.395 \AA$, which is only $0.5 \%$ smaller than the experimental lattice constant of $a=4.419 \AA$. However the half-metallic DOS, BS, and gap from the GGA are similar to those from the LSDA calculations. The GGA spin moments of both $\mathrm{Cr}$ and $\mathrm{O}$ are slightly larger, whereas the GGA orbital moment of $\mathrm{O}$ is similar to the LSDA moment of $\mathrm{O}$, and the moment of $\mathrm{Cr}$ from the GGA is slightly smaller than that from the LSDA. These results indicate that the on-site Coulomb correlations $(U)$ rather than the gradient corrections to the exchange-correlation potential, are more important for

TABLE II. Calculated spin and orbital magnetic moments $\left(\mu_{B} /\right.$ atom) and the ratio $L / S(\%)$ of $\mathrm{Cr}$ and $\mathrm{O}$ in $\mathrm{CrO}_{2}$ from the LSDA $(U=J=0 \mathrm{eV})$ and from the LSDA $+U$. The on-site Coulomb energy $U$ is in units of $\mathrm{eV}$ and the exchange parameter $J=0.87 \mathrm{eV}$.

\begin{tabular}{|c|c|c|c|c|c|c|}
\hline $\begin{array}{c}U \\
(\mathrm{eV})\end{array}$ & $\begin{array}{c}\mathrm{Cr} \\
\text { spin } \\
\left(\mu_{B}\right)\end{array}$ & $\begin{array}{c}\text { Orbital } \\
\left(\mu_{B}\right)\end{array}$ & $\begin{array}{l}L / S \\
(\%)\end{array}$ & $\begin{array}{c}\mathrm{O} \\
\text { spin } \\
\left(\mu_{B}\right)\end{array}$ & $\begin{array}{c}\text { Orbital } \\
\left(\mu_{B}\right)\end{array}$ & $\begin{array}{l}L / S \\
(\%)\end{array}$ \\
\hline 0 & 1.89 & -0.037 & -2.0 & -0.042 & -0.0011 & 2.6 \\
\hline 2 & 1.94 & -0.046 & -2.4 & -0.058 & -0.0019 & 3.2 \\
\hline 3 & 1.99 & -0.051 & -2.6 & -0.079 & -0.0025 & 3.2 \\
\hline 4 & 2.03 & -0.056 & -2.8 & -0.094 & -0.0030 & 3.2 \\
\hline 6 & 2.08 & -0.067 & -3.2 & -0.111 & -0.0040 & 3.6 \\
\hline 9 & 2.12 & -0.083 & -3.9 & -0.124 & -0.0047 & 3.8 \\
\hline
\end{tabular}


TABLE III. Calculated spin and orbital magnetic moments $\left(\mu_{B} /\right.$ atom) and the ratio $L / S(\%)$ of $\mathrm{Cr}$ and $\mathrm{O}$ for [001] and [100] magnetization in $\mathrm{CrO}_{2}$ from $\operatorname{LSDA}(U=J=0 \mathrm{eV})$ and $\mathrm{LSDA}+U(U=3 \mathrm{eV}, J=0.87 \mathrm{eV})$.

\begin{tabular}{|c|c|c|c|c|c|c|c|}
\hline $\begin{array}{c}U \\
(\mathrm{eV})\end{array}$ & & $\begin{array}{c}\mathrm{Cr} \\
\text { spin } \\
\left(\mu_{B}\right)\end{array}$ & $\begin{array}{c}\text { Orbital } \\
\left(\mu_{B}\right)\end{array}$ & $\begin{array}{l}L / S \\
(\%)\end{array}$ & $\begin{array}{c}\mathrm{O} \\
\text { spin } \\
\left(\mu_{B}\right)\end{array}$ & $\begin{array}{c}\text { Orbital } \\
\left(\mu_{B}\right)\end{array}$ & $\begin{array}{l}L / S \\
(\%)\end{array}$ \\
\hline 0 & [001] & 1.89 & -0.037 & -2.0 & -0.042 & -0.0011 & 2.6 \\
\hline 0 & {$[100]$} & 1.89 & -0.034 & -1.8 & -0.042 & -0.0000 & 0.0 \\
\hline 3 & [001] & 1.99 & -0.051 & -2.6 & -0.079 & -0.0025 & 3.2 \\
\hline 3 & {$[100]$} & 1.99 & -0.050 & -2.5 & -0.079 & -0.0004 & 0.5 \\
\hline
\end{tabular}

the improved band dispersions of the chromium dioxide.

The calculated spin and orbital magnetic moments and the orbital to spin moment ratio $L / S$ of $\mathrm{Cr}$ and $\mathrm{O}$ for [001] magnetization are summarized in Table II. The LSDA ( $U$ $=0 \mathrm{eV})$ gives spin moments of 1.89 and $-0.042 \mu_{B} /$ atom for $\mathrm{Cr}$ and $\mathrm{O}$, respectively. The spin moment of the $\mathrm{Cr}$ atoms is antiparallel to that of the $\mathrm{O}$ atoms. The orbital moment of $\mathrm{Cr}$ is also antiparallel to the spin moment of $\mathrm{Cr}$, being consistent with Hund's rules for $3 d$ shells less than half full. In contrast, the orbital moment of the $\mathrm{O}$ atoms is parallel to the $\mathrm{O}$ spin moment because the $\mathrm{O}-2 p$ shell is more than half filled. Hence the orbital moment of $\mathrm{O}$ is parallel to that of $\mathrm{Cr}$, and the resultant magnetic moments of $\mathrm{Cr}$ and $\mathrm{O}$ are antiferromagnetically coupled in $\mathrm{CrO}_{2}$. As usually happened in the LSDA calculations, the obtained orbital moments of -0.037 and $-0.0011 \mu_{B} /$ atom for $\mathrm{Cr}$ and $\mathrm{O}$, respectively, are both too small as compared with the corresponding experimental moments. ${ }^{9}$ Note that the magnitude of $L / S$ of oxygen is larger than that of $\mathrm{Cr}$, indicating the relatively significant orbital moment of oxygen in $\mathrm{CrO}_{2}$ as compared with that in other transition metal oxides.

Also shown in Table II are the spin and orbital magnetic moments from the LSDA $+U$ calculations. The spin moments of both $\mathrm{Cr}$ and $\mathrm{O}$ are enhanced because the on-site Coulomb energy $U$ further localizes the $3 d$ orbital. For the literature values of $U=3 \mathrm{eV}$ and $J=0.87 \mathrm{eV}^{12}$ considered, the spin moments of $\mathrm{Cr}$ and $\mathrm{O}$ are raised by an amount of 0.10 and $0.037 \mu_{B} /$ atom, respectively. Meanwhile, the orbital moments of $\mathrm{Cr}$ and $\mathrm{O}$ increases dramatically up to -0.051 and $-0.0025 \mu_{B}$ /atom, respectively. The obtained orbital moments from the LSDA $+U$ are in good agreement with the experimental orbital moments of -0.05 and $-0.003 \mu_{B}$ /atom, respectively. ${ }^{9}$ For larger $U$ considered, both spin moments of $\mathrm{Cr}$ and $\mathrm{O}$ increase more or less linearly with respective to $U$, while the half-metallic property with a total magnetic moment of $2 \mu_{B} / \mathrm{fu}$ remains unchanged. Note that increasing $U$ reduces the total charges in the Cr muffin-tin spheres and increases the total charges in the $\mathrm{O}$ muffin-tin spheres, thus increasing the ionicity of both the $\mathrm{Cr}$ and $\mathrm{O}$ atoms. Since larger $U$ suppresses the octahedral ligand fields on the $\mathrm{Cr}$ atoms by strongly localizing the $3 d$ electrons, the orbital moments of the $\mathrm{Cr}$ and $\mathrm{O}$ are thus raised further by increasing $U$ (see Table II). Similar trends have been found in $\mathrm{CoO}^{7}$ and $\mathrm{NiO}^{8}$ Nevertheless, the orbital magnetic moment of $\mathrm{Cr}$ is found to be quenched in $\mathrm{CrO}_{2}$, in contrast to the large orbital magnetic moments found in $\mathrm{CoO}^{5}$ and $\mathrm{NiO}^{6}$ In this work, different values of $J$ from 0.3 up to $1.5 \mathrm{eV}$ were also used for $U=2,3$, and $4 \mathrm{eV}$ cases. It is found that the spin moments of $\mathrm{Cr}$ and $\mathrm{O}$ are suppressed by larger values of $J$, whereas the orbital moments are not sensitive to $J$.

Table III shows the spin and orbital moment and the $L / S$ ratio of $\mathrm{Cr}$ and $\mathrm{O}$ in $\mathrm{CrO}_{2}$ for [001] and [100] directions of magnetization from the LSDA and from the LSDA $+U$ with $U=3 \mathrm{eV}$ and $J=0.87 \mathrm{eV}$. The most visible feature is the large anisotropy of the orbital moments of O. In the [100] magnetization, the orbital moment of $\mathrm{O}$ is destroyed from the LSDA calculations, while it is strongly suppressed in the LSDA $+U$ approach. Meanwhile, both the LSDA and the LSDA $+U$ give slightly smaller orbital moments for $\mathrm{Cr}$. On the other hand, the anisotropy in the spin moments of $\mathrm{Cr}$ and $\mathrm{O}$ are negligibly small from both the LSDA and the LSDA+ $U$ calculations. The anisotropy in the orbital magnetic moments indicates that the [001] direction of magnetization, which provides larger orbital moments of $\mathrm{Cr}$ and $\mathrm{O}$, would be the easy axis of $\mathrm{CrO}_{2}$. The smaller anisotropy of the orbital moment of $\mathrm{Cr}$ from the LSDA $+U$ calculations also implies that the LSDA $+U$, rather than the LSDA, would give a smaller magnetocrystalline anisotropy energy for $\mathrm{CrO}_{2}$.

TABLE IV. Calculated spin and orbital magnetic moments $\left(\mu_{B} /\right.$ atom) and the ratio $L / S(\%)$ of $\mathrm{Cr}$ and $\mathrm{O}$ in $\mathrm{CrO}_{2}$ with respect to lattice expansion from LSDA.

\begin{tabular}{ccccccc}
\hline \hline & $\begin{array}{c}\mathrm{Cr} \\
\text { spin } \\
\left(\mu_{B}\right)\end{array}$ & $\begin{array}{c}\text { Orbital } \\
\left(\mu_{B}\right)\end{array}$ & $\begin{array}{c}L / S \\
(\%)\end{array}$ & $\begin{array}{c}\text { O } \\
\text { spin } \\
\left(\mu_{B}\right)\end{array}$ & $\begin{array}{c}\text { Orbital } \\
\left(\mu_{B}\right)\end{array}$ & $\begin{array}{c}L / S \\
(\%)\end{array}$ \\
\hline 0.90 & 1.80 & -0.033 & -1.8 & -0.010 & -0.0009 & 9.0 \\
0.95 & 1.85 & -0.035 & -1.9 & -0.025 & -0.0010 & 4.0 \\
1.00 & 1.89 & -0.037 & -2.0 & -0.042 & -0.0011 & 2.6 \\
1.05 & 1.94 & -0.038 & -2.0 & -0.059 & -0.0012 & 2.0 \\
1.10 & 1.99 & -0.040 & -2.0 & -0.078 & -0.0013 & 1.7 \\
1.15 & 2.04 & -0.041 & -2.0 & -0.099 & -0.0015 & 1.5 \\
\hline \hline
\end{tabular}


TABLE V. Distances (D) between the transition metal atoms and octahedrally coordinated oxygen atoms (nearest neighbors) in transition metal oxides (in atomic units).

\begin{tabular}{cccccc}
\hline \hline & $\begin{array}{c}\mathrm{NiO} \\
\mathrm{Ni}-\mathrm{O}\end{array}$ & $\begin{array}{c}\mathrm{CoO} \\
\mathrm{Co}-\mathrm{O}\end{array}$ & $\begin{array}{c}\mathrm{FeO} \\
\mathrm{Fe}-\mathrm{O}\end{array}$ & $\begin{array}{c}\mathrm{Fe}_{3} \mathrm{O}_{4} \\
\mathrm{Fe}(\mathrm{B})-\mathrm{O}\end{array}$ & $\begin{array}{c}\mathrm{CrO}_{2} \\
\mathrm{Cr}-\mathrm{O}\end{array}$ \\
\hline $\mathrm{D}\left(a_{0}\right)$ & 3.947 & 4.025 & 4.095 & 3.891 & 3.579 \\
\hline \hline
\end{tabular}

The LSDA spin and orbital magnetic moments and the ratio $L / S$ of $\mathrm{Cr}$ and $\mathrm{O}$ as a function of volume from $0.9 V_{0}$ to $1.15 V_{0}$ for $\mathrm{CrO}_{2}$ are listed in Table IV. $V_{0}$ is the experimental volume. Both the spin and orbital moments of $\mathrm{Cr}$ and $\mathrm{O}$ increase monotonically with increasing volume. This could be attributed to the reduction of the crystal fields and the hybridizations between $\mathrm{Cr}-3 d$ and $\mathrm{O}-2 p$ bands caused by lattice expansion. Interestingly, the increasing rate are quite different for the spin and orbital moments of $\mathrm{Cr}$ and $\mathrm{O}$. As shown in Table IV, the magnitude of $L / S$ for $\mathrm{Cr}$ increases very slowly with increasing $V / V_{0}$, whereas that for $\mathrm{O}$ decreases significantly. This suppression of $L / S$ of $\mathrm{O}$ is due to the faster growth with increasing volume of the $\mathrm{O}$ spin moment compared with the $\mathrm{O}$ orbital moment, demonstrating a relatively stronger dependence of spin moment of $\mathrm{O}$ on lattice volume. Note that the spin and orbital moments are both zero in bcc $\mathrm{Cr}$, which reveals that the $3 d$ electrons are much more localized in $\mathrm{CrO}_{2}$ than in $\mathrm{Cr}$ metal. However, for all the lattice volumes considered, the volumes are not small enough to quench all the moments, and are not large enough to give large orbital moments as that in $\mathrm{CoO}^{7}$ and in $\mathrm{NiO}^{8}$

The unit cell of $\mathrm{CrO}_{2}$ is relatively compact in the sense that the distance between the nearest $\mathrm{Cr}-\mathrm{O}$ neighbors is short and the size of the $\mathrm{Cr}-3 d$ orbital is large, as compared with those in other transition metal oxides such as $\mathrm{NiO}, \mathrm{CoO}$, $\mathrm{FeO}$, and $\mathrm{Fe}_{3} \mathrm{O}_{4}$. For all the above-mentioned materials, the transition metal atoms, including the octahedral $B$-site $\mathrm{Fe}$ in $\mathrm{Fe}_{3} \mathrm{O}_{4}$, are surrounded by oxygen octahedra except the tetrahedral $A$-site $\mathrm{Fe}$ in $\mathrm{Fe}_{3} \mathrm{O}_{4}$. The nearest-neighbor distances between the transition metal atoms and the oxygen atoms in $\mathrm{NiO}, \mathrm{CoO}, \mathrm{FeO}, \mathrm{Fe}_{3} \mathrm{O}_{4}\left(B\right.$-site), and $\mathrm{CrO}_{2}$ are listed in Table $\mathrm{V}$. They are quite similar in these oxides, except in $\mathrm{CrO}_{2}$ where the distance is $8 \% \sim 13 \%$ shorter than that in the other compounds. This indicates a much stronger hybridization between the $\mathrm{Cr}-3 d$ and $\mathrm{O}-2 p$ orbitals in $\mathrm{CrO}_{2}$, thus revealing the reason why the orbital magnetic moments of the transition metal atoms are much more strongly quenched in $\mathrm{CrO}_{2}$ than in other oxides. This notion is further strengthened by comparison of the charge density distributions of the $3 d$ orbitals in these transition metal atoms. Table VI shows the

TABLE VI. Expectation values of $r$ and $r^{2}$ of O $2 p$ and transition metal atom $3 d$ orbitals from the atomic SIC-LSDA calculations (in atomic units).

\begin{tabular}{ccccccc}
\hline \hline$\left(a_{0}\right)$ & & $\mathrm{Ni}-3 d$ & $\mathrm{Co}-3 d$ & $\mathrm{Fe}-3 d$ & $\mathrm{Cr}-3 d$ & $\mathrm{O}-2 p$ \\
\hline$\langle r\rangle$ & $\uparrow$ & 0.969 & 1.020 & 1.080 & 1.400 & 1.201 \\
$\langle r\rangle$ & $\downarrow$ & 0.998 & 1.070 & 1.157 & & 1.294 \\
$\left\langle r^{2}\right\rangle$ & $\uparrow$ & 1.242 & 1.370 & 1.531 & 2.675 & 1.882 \\
$\left\langle r^{2}\right\rangle$ & $\downarrow$ & 1.333 & 1.536 & 1.807 & & 2.221 \\
\hline \hline
\end{tabular}

expectation values $\langle r\rangle$ and $\left\langle r^{2}\right\rangle$ of $\mathrm{O} 2 p$ and transition metal atom $3 d$ orbitals from the atomic self-interaction corrected LSDA (SIC-LSDA) calculations. ${ }^{20}$ The details of the computational method are described in Ref. 21. Note that the SICLSDA scheme ${ }^{20}$ for the atomic systems, which eliminates the spurious electron self-interactions in the LSDA, is akin to the LSDA $+U$ approach for the solid systems, which includes the on-site Coulomb interactions in the localized $d$ and $f$ orbitals. Without the electron self-interactions, the SICLSDA provides a more localized charge density distribution for an atomic system as compared with that from the LSDA. The $s$-rich atomic configurations for $3 d$ transition metal atoms, namely $4 s^{2} 3 d^{n}$, were used in the atomic SIC-LSDA calculations, and the spin alignments were arranged in accordance with the Hund's rules. As shown in Table VI, $\langle r\rangle$ of $3 d$ orbital is larger for the atoms with a smaller atomic number. The $\mathrm{Cr}-3 d$ orbital is thus located at a relatively outer region with the expectation value of $r$ being 21\% 44\% larger than that of $3 d$ orbitals in the other transition metal atoms. Further, $\left\langle r^{2}\right\rangle$ of $\mathrm{Cr}-3 d$ orbital is strongly enhanced, indicating a much more diffusive $3 d$ orbital. Thus, in addition to the much smaller distances between the $\mathrm{Cr}$ and $\mathrm{O}$ atoms in $\mathrm{CrO}_{2}$ (Table V) which would cause a stronger transition metal-oxygen hybridization, the octahedral ligand field on $\mathrm{Cr}$ in $\mathrm{CrO}_{2}$ is also much stronger than that on the other transition metal atoms in the other oxides. The orbital magnetic moment of $\mathrm{Cr}$ in $\mathrm{CrO}_{2}$ is therefore quenched by the strong Cr-3d and $\mathrm{O}-2 p$ hybridization and strong ligand crystal field.

\section{CONCLUSIONS}

In conclusion, we have performed self-consistent FPLMTO calculations for ferromagnetic half-metal $\mathrm{CrO}_{2}$ within both the LSDA and the LSDA $+U$ schemes. The onsite Coulomb energy $U$ strongly affects the DOS and BS. For $U=3 \mathrm{eV}$ and $J=0.87 \mathrm{eV}$, the gap in the minority spin at the Fermi energy is enhanced and the exchange splitting is raised to $4.22 \mathrm{eV}$ which is compatible with about $4.5 \mathrm{eV}$ from the LMTO-ASA calculations ${ }^{12}$ and about $5 \mathrm{eV}$ from the experiments. ${ }^{13}$ It is found that the on-site Coulomb interaction $U$ dramatically enhanced the spin and orbital moments. For $U=3 \mathrm{eV}$ and $J=0.87 \mathrm{eV}$, the calculated orbital moments of $\mathrm{Cr}$ and $\mathrm{O}$ are -0.051 and $-0.0025 \mu_{B} /$ atom, respectively, in good agreement with the experimental moments of $-0.05 \mu_{B} /$ atom for $\mathrm{Cr}$ and $-0.003 \mu_{B} /$ atom for $\mathrm{O}$ from XMCD measurements. ${ }^{9}$

\section{ACKNOWLEDGMENTS}

The authors would like to thank C. S. Hsue and D. J. Huang for stimulating discussions. This work was supported by the National Science Council of Taiwan (NSC90-2119-M007-004, NSC90-2112-M-002-040).

\footnotetext{
${ }^{1}$ M. Imada, A. Fujimori, and Y. Tokura, Rev. Mod. Phys. 70, 1039 (1998).

${ }^{2}$ V. I. Anisimov, J. Zaanen, and O. K. Andersen, Phys. Rev. B 44, 943 (1991).

${ }^{3}$ V. I. Anisimov, F. Aryasetiawan, and A. I. Liechtenstein, J. Phys.: Condens. Matter 9, 767 (1997)

${ }^{4}$ B. L. Chamberland, Crit. Rev. Solid State Mater. Sci. 7, 1 (1977).
} 
${ }^{5}$ D. C. Khan and R. A. Erickson, Phys. Rev. B 1, 2243 (1970).

${ }^{6}$ V. Fernandez, C. Vettier, F. de Bergevin, C. Giles, and W. Neubeck, Phys. Rev. B 57, 7870 (1998).

${ }^{7}$ I. V. Solovyev, A. I. Liechtenstein, and K. Terakura, Phys. Rev. Lett. 80, 5758 (1998).

${ }^{8}$ S. K. Kwon and B. I. Min, Phys. Rev. B 62, 73 (2000).

${ }^{9}$ D. J. Huang, H. T. Jeng, C. F. Chang, G. Y. Guo, J. Chen, W. P. Wu, S. C. Chung, S. G. Shyu, C. C. Wu, H. J. Lin, and C. T. Chen, Phys. Rev. B (to be published).

${ }^{10}$ K. Schwarz, J. Phys. F: Met. Phys. 16, L211 (1986).

${ }^{11}$ S. P. Lewis, P. B. Allen, and T. Sasaki, Phys. Rev. B 55, 10253 (1997).

${ }^{12}$ M. A. Korotin, V. I. Anisimov, D. I. Khomskii, and G. A. Sawatzky, Phys. Rev. Lett. 80, 4305 (1998).
${ }^{13}$ T. Tsujioka, T. Mizokawa, J. Okamoto, A. Fijimori, M. Nohara, H. Takagi, K. Yamaura,and M. Takano, Phys. Rev. B 56, R15509 (1997).

${ }^{14}$ C. B. Stagarescu, X. Su, D. E. Eastman, K. N. Altmann, F. J. Himpsel, and A. Gupta, Phys. Rev. B 61, R9233 (2000).

${ }^{15}$ I. I. Mazin, D. J. Singh, and C. A. Draxl, Phys. Rev. B 59, 411 (1999).

${ }^{16}$ S. Y. Savrasov, Phys. Rev. B 54, 16470 (1996).

${ }^{17}$ A. I. Liechtenstein, V. I. Anismov, and J. Zaanen, Phys. Rev. B 52, R5467 (1995).

${ }^{18}$ S. H. Vosko, L. Wilk, and M. Nusair, Can. J. Phys. 58, 1200 (1980).

${ }^{19}$ P. Porta, M. Marezio, J. P. Remeika, and P. D. Dernier, Mater. Res. Bull. 7, 157 (1972).

${ }^{20}$ J. P. Perdew and A. Zunger, Phys. Rev. B 23, 5048 (1981).

${ }^{21}$ H. T. Jeng and C. S. Hsue, Chin. J. Phys. (Taipei) 35, 215 (1997). 